\title{
A New Algorithm for Solving Large-Scale Generalized Eigenvalue Problem Based on Projection Methods
}

\author{
F. Abbasi Nedamani, A. H. Refahi Sheikhani $(\mathbb{D}$, and H. Saberi Najafi \\ Department of Applied Mathematics, Faculty of Mathematical Sciences, Lahijan Branch, Islamic Azad University, Lahijan, Iran \\ Correspondence should be addressed to A. H. Refahi Sheikhani; ah_refahi@yahoo.com
}

Received 17 September 2020; Revised 10 November 2020; Accepted 29 November 2020; Published 14 December 2020

Academic Editor: Li-Tao Zhang

Copyright ( $\odot 2020$ F. Abbasi Nedamani et al. This is an open access article distributed under the Creative Commons Attribution License, which permits unrestricted use, distribution, and reproduction in any medium, provided the original work is properly cited.

In this paper, we consider four methods for determining certain eigenvalues and corresponding eigenvectors of large-scale generalized eigenvalue problems which are located in a certain region. In these methods, a small pencil that contains only the desired eigenvalue is derived using moments that have obtained via numerical integration. Our purpose is to improve the numerical stability of the moment-based method and compare its stability with three other methods. Numerical examples show that the block version of the moment-based (SS) method with the Rayleigh-Ritz procedure has higher numerical stability than respect to other methods.

\section{Introduction}

Many problems arising in different fields of science and engineering can be reduced to the generalized eigenvalue problem [1-3]:

$$
A x=\lambda B x,
$$

where $A, B$ are $n \times n$ real or complex, large, sparse, and only a few of the eigenvalues are desired. Also, when is $B=I$ (identity matrix), we have a standard eigenvalue problem. Computing eigenpairs $(\lambda, x)$ of the generalized and standard eigenvalue problems is one of the important problems in many scientific applications [4-7]. There are several methods for solving such eigenvalue problems [8]. Among these methods, the iterative methods are used to generate a subspace that contains the desired eigenvectors. Techniques based on the Krylov subspaces are powerful tools for building desired subspaces for large-scale eigenvalue problems [9-11]. Expressed methods in this article find all of the zeros that lie in a circle using numerical integration. In this paper, we briefly describe moment-based method in Section 2, Rayleigh-Ritz with contour integral method in Section 3, block version of the Sakurai-Sugiura method in Section 4, and block version of the SS method with
Rayleigh-Ritz procedure in Section 5 for solving generalized eigenvalue problem (1). In Section 6, we provide four numerical tests for comparing four methods, and in Section 6, we apply the BSSRR method with selected matrices from different application areas, and finally, we draw some conclusions in Section 7.

\section{Moment-Based Method (SS Method)}

For solving (1), we consider computing entire poles of a rational function:

$$
f(z)=u^{H}(z B-A)^{-1}, \quad v, u, v \in \mathbb{C}^{n} /\{0\} .
$$

Those are eigenvalues $\lambda$ of equation (1) and lie in a circle using numerical integration. Let $\Gamma$ be positively oriented closed Jordan curve [12] in the complex plane and $\lambda_{1}, \ldots, \lambda_{n}$ be distinct eigenvalues that lie in the $\Gamma$. Let

$$
\mu_{k}=\frac{1}{2 \pi i} \int_{\Gamma}^{-}(z-\gamma)^{k} f(z) \mathrm{d} z, \quad k=0,1, \ldots,
$$

where $\gamma$ is located inside $\Gamma$ and the $m \times m$ Hankel matrices $H_{m}, H_{m}^{<}$be $H_{m}=\left[\mu_{i+j-2}\right]_{i, j=1}^{m}$ and $H_{m}^{<}=\left[\mu_{i+j-1}\right]_{i, j=1}^{m}$. Also, let 
$s_{k}=\frac{1}{2 \pi i} \int_{\Gamma}^{-}(z-\gamma)^{k}(z B-A)^{-1} v \mathrm{~d} z, \quad k=0,1, \ldots$

Then, we have the following theorem.

Theorem 1. If $v_{j}=0$ for $1 \leq j \leq m$, then the eigenvalues of the pencil $H_{m}^{<}-\lambda H_{m}$ are given by $\lambda_{1}-\gamma, \ldots, \lambda_{m}-\gamma$.

Proof. In [13], by approximating the integral of equation (3) via the $\mathrm{N}$-point trapezoidal rule, we obtain

$$
\mu_{k} \approx \widehat{\mu}_{k}=\frac{1}{N} \sum_{j=0}^{N-1}\left(\omega_{j}-\gamma\right)^{k+1} f\left(\omega_{j}\right), \quad k=0,1, \ldots
$$

Let $\xi_{1}, \ldots, \xi_{m}$ be the eigenvalues of pencil $H_{m}^{<}-\lambda H_{m}$. We regard $\hat{\lambda}_{j}=\gamma+\xi_{j}, 1 \leq j \leq m$ as the approximations for $\widehat{\lambda}_{1}, \ldots, \widehat{\lambda}_{m}$ and

$$
\begin{aligned}
\left(\omega_{j} B-A\right) y_{j} & =v, \quad j=0,1, \ldots, N-1, \\
\widehat{s}_{k} & =\frac{1}{N} \sum_{j=0}^{N-1}\left(\omega_{j}-\gamma\right)^{k+1} y_{j}, \quad k=0,1, \ldots
\end{aligned}
$$

Also, let $\widehat{V}_{m}$ be the Vandermonde matrix for $\xi_{1}, \ldots, \xi_{m}$. Then, the approximations for the eigenvectors are obtained by

$$
\left[\widehat{q}_{1}, \ldots, \widehat{q}_{m}\right]=\left[\widehat{s}_{0}, \ldots, \widehat{s}_{m-1}\right] \widehat{V}_{m}^{-T}
$$

\section{Rayleigh-Ritz with Contour Integral Method (CIRR Method)}

We consider (1), let $A, B \in \mathbb{R}^{n \times n}$ be symmetric and let $\mathrm{B}$ be positive definite and $\left(\lambda_{j}, x_{j}\right), 1 \leq j \leq n$ be eigenpairs of the matrix pencil $(A, B)$. We apply a Rayleigh-Ritz procedure with an orthonormal basis $Q \in \mathbb{R}^{n \times m}$. The projected matrices are given by $\widetilde{A}=Q^{T} A Q$ and $\widetilde{B}=Q^{T} B Q \cdot Q \in \mathbb{R}^{n \times m}$ is used to generate a sequence of subspace containing approximations to the desired eigenvector. The Ritz values of the projected pencil $(\widetilde{A}, t \widetilde{B})$ are taken as approximate eigenvalues for original pencil $(A, B)$ with corresponding Ritz vectors. In this method, by applying the Rayleigh-Ritz procedure moments are not explicitly used [17]. The algorithm is as follows.

Rayleigh-Ritz procedure

(1) Construct an orthonormal basis $Q$

(2) Form $\widetilde{A}=Q^{T} A Q$ and $\widetilde{B}=Q^{T} B Q$

(3) Compute the eigenpairs $\left(\theta_{j}, \omega_{j}\right) \quad(1 \leq j \leq m)$ of $(\widetilde{A}, t \widetilde{B})$

(4) Set $p_{j}=Q \omega_{j}, j=1, \ldots, M$

Theorem 2. Let $s_{k}$ be defined by (4). Suppose that vis expanded by the eigenvectors $\left\{x_{1}, \ldots, x_{n}\right\}$ as

$$
v=\sum_{j=1}^{n} \alpha_{j} x_{j}
$$

Then,

$$
s_{k}=\sum_{j=1}^{m} \alpha_{j}\left(\lambda_{j}-\gamma\right)^{k} x_{j}, \quad j=0,1, \ldots, m-1 .
$$

Proof. It follows from (4) and (8) that

$$
s_{k}=\frac{1}{2 \pi i} \int_{\Gamma}^{-} \sum_{j=1}^{n} \alpha_{j}(z-\gamma)^{k}(z B-A)^{-1} B x_{j} \mathrm{~d} z .
$$

Since $\left(\lambda_{j}, x_{j}\right)$ is an eigenpair of the matrix pencil $(A, B)$, we have $(z B-A) x_{j}=\left(z-\lambda_{j}\right) B x_{j}$ and thus $(z B-A)^{-1} B x_{j}=\left(z-\lambda_{i}\right)^{-1} x_{j}$, and thus

$$
s_{k}=\sum_{j=1}^{n} \frac{1}{2 \pi i} \int_{\Gamma}^{-} \frac{\alpha_{j}(z-\gamma)^{k}}{z-\lambda_{j}} x_{j} \mathrm{~d} z, \quad k=0,1, \ldots, m-1 .
$$

By the residue theorem, we obtain the result.

We define the $m \times m$ Vandermonde matrix with $\lambda_{1}-$ $\gamma, \ldots, \lambda_{m}-\gamma$ by

$$
V=\left[\begin{array}{cccc}
1 & \left(\lambda_{1}-\gamma\right) & \ldots & \left(\lambda_{1}-\gamma\right)^{m-1} \\
1 & \left(\lambda_{2}-\gamma\right) & \ldots & \left(\lambda_{2}-\gamma\right)^{m-1} \\
\vdots & \vdots & \vdots & \vdots \\
1 & \left(\lambda_{m}-\gamma\right) & \ldots & \left(\lambda_{m}-\gamma\right)^{m-1}
\end{array}\right]
$$

From the equation (9), we have

$$
S=X D V \text {, }
$$

where $\quad S=\left[s_{0}, \ldots, s_{m-1}\right], X=\left[x_{1}, \ldots, x_{m}\right], \quad$ and $D=\operatorname{diag}\left(\alpha_{1}, \ldots, \alpha_{m}\right)$.

Theorem 3. If $\lambda_{1}, \ldots, \lambda_{m}$ are distinct and $\alpha_{j} \neq 0$ for $1 \leq j \leq m$, then

$$
\operatorname{span}\left\{q_{1}, \ldots, q_{m}\right\}=\operatorname{span}\left\{x_{1}, \ldots, x_{m}\right\}
$$

Proof. Since $\lambda_{1}, \ldots, \lambda_{m}$ are mutually distinct and $\alpha_{j} \neq 0$ for $1 \leq j \leq m$. V and $\mathrm{D}$ are nonsingular. Therefore, it follows from (13) that

$$
\operatorname{span}\left\{s_{0}, \ldots, s_{m-1}\right\}=\operatorname{span}\left\{x_{1}, \ldots, x_{m}\right\} .
$$

Since the vectors $\left\{q_{1}, \ldots, q_{m}\right\}$ are orthonormal basis of $\operatorname{span}\left\{s_{0}, \ldots, s_{m-1}\right\}$, equation (14) holds.

For nonzero vector $v \in \mathbb{R}^{n}$, we define the moments:

$$
\mu_{k}=\frac{1}{2 \pi i} \int_{\Gamma}^{-}(z-\gamma)^{k}(B v)^{T}(z B-A)^{-1} B v \mathrm{~d} z, \quad k=0,1, \ldots,
$$

where $\gamma$ is located inside $\Gamma$. Also, we obtain the following approximations via the $\mathrm{N}$-point trapezoidal rule: 


$$
\begin{aligned}
\mu_{k} \simeq \widehat{\mu}_{k} & =\frac{1}{N} \sum_{j=0}^{N-1}\left(\omega_{j}-\gamma\right)^{k+1}(B v)^{T}\left(\omega_{j} B-A\right)^{-1} B v, \quad k=0,1, \ldots, \\
\widehat{s}_{k} & =\frac{1}{N} \sum_{j=0}^{N-1}\left(\omega_{j}-\gamma\right)^{k+1}\left(\omega_{j} B-A\right)^{-1} B v, \quad k=0,1, \ldots
\end{aligned}
$$

\section{Block Sakurai-Sugiura Method (BSS Method)}

In this method, for solving (1), we reformulate the SS method in the context of the resolvent theory. This method has the potential to resolve degenerated eigenvalues.

Theorem 4. Let $z B-A$ be a regular pencil of order $N$. Then, there exist nonsingular matrices $\widetilde{P}, Q \in \mathbb{C}^{N \times N}$ such that

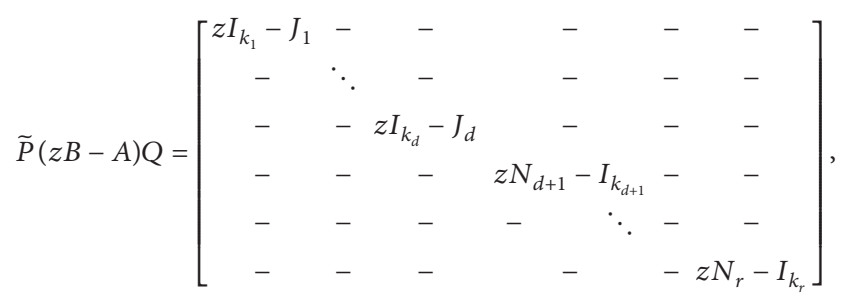

where $J_{i}, N_{i} \in \mathbb{C}^{k_{i} \times k_{i}}$ are Jordan blocks, $N_{i}$ is nilpotent, and $I_{k}$ denotes the identity matrix of order $k$.

Proof. In [12].

Here, because $\widetilde{P}, Q$ are the regular matrices, we can define $P=\widetilde{P}^{-1}$ and $\widetilde{Q}=Q^{-1}$. According to the (18), we will partition row vectors in $\widetilde{P}$ and $\widetilde{Q}$ into $\widetilde{P}_{i}, \widetilde{Q}_{i} \in \mathbb{C}^{k_{i} \times N}$, and column vectors in $P$ and $Q$ into $P_{i}, Q_{i} \in \mathbb{C}^{N \times k_{i}}$, respectively, for $i=1, \ldots, r$.

Theorem 5. Resolvent of the regular pencil $(z B-A)^{-1}$ is decomposed into

$$
(z B-A)^{-1}=\sum_{i=1}^{d} Q_{i}\left\{\sum_{m=0}^{k_{i}-1} \frac{\left(J_{i}-\alpha_{i} I_{k_{k}}\right)^{m}}{\left(z-\alpha_{i}\right)^{m+1}}\right\} \widetilde{P}_{i}-\sum_{i=d+1}^{r} Q_{i}\left\{\sum_{m=0}^{k_{i}-1} z^{m} N_{i}^{m}\right\} \widetilde{P}_{i},
$$

where $\alpha_{i}$ is an eigenvalue of Jordan block $J_{i}$.

Proof. Let $W=\widetilde{P}(z B-A) Q$. According to Theorem 4, we have

$$
\begin{aligned}
(z B-A)^{-1}= & Q W^{-1} \widetilde{P}=\sum_{i=1}^{d} Q_{i}\left(z I_{k_{i}}-J_{i}\right)^{-1} \widetilde{P}_{i} \\
& +\sum_{i=d+1}^{r} Q_{i}\left(z N_{i}-I_{k_{i}}\right)^{-1} \widetilde{P}_{i} .
\end{aligned}
$$

Using the resolvent of the Jordan block,

$$
R\left(z, J_{i}\right)=\left(z I_{k_{i}}-J_{i}\right)^{-1}=\sum_{m=0}^{k_{i}-1} \frac{\left(J_{i}-\alpha_{i} I_{k_{i}}\right)^{m}}{\left(z-\alpha_{i}\right)^{m+1}}
$$

And $\left(z N_{i}-I_{k_{i}}\right)^{-1}=-z^{-1} R\left(z^{-1}, N_{i}\right)$, we get the result.
Theorem 6. The localized moment matrix is written as

$$
M_{n}=\sum_{i ; \alpha_{i} \in G} Q_{i} J_{i}^{n} \widetilde{Q}_{i}
$$

Proof. In [18].

Definition 1. Let $\mathrm{C}$ and $\mathrm{D}$ be arbitrary $N \times m$ matrices, where $N>m \geq k_{\Gamma}$. A size-reduced moment matrix is defined as

$$
M_{n}=C^{H} M_{n} D \in \mathbb{C}^{m \times m} .
$$

Theorem 7. If ranks of both $C^{H} Q_{\Gamma}$ and $\widetilde{Q}_{\Gamma} D$ are $k_{\Gamma}$, nonsingular part of a matrix pencil $z M_{0}-M_{1}$ is equivalent to $z I_{k_{T}}-J_{\Gamma}$.

Proof. In [18].

Theorem 8. The right eigenvectors of the original matrix pencil $z B-A$ are given by $Q_{\Gamma}=M_{0} D Q_{\Gamma}$, and its adjoint is given by $\widetilde{\mathrm{Q}}_{\Gamma}=\widetilde{P}_{\Gamma} C^{H} M_{0}$.

Proof. In [18].

Theorem 9. If all elements of $\widetilde{\mathrm{Q}}_{\Gamma} v$ and $v^{H} \mathrm{Q}_{\Gamma}$ are nonzero, and there is no degeneracy in $J_{\Gamma}$, then nonsingular part of a matrix pencil $z H_{m}-H_{m}^{<}$is equivalent to $z I_{k_{\Gamma}}-J_{\Gamma}$.

Proof. By choosing row vectors of $C^{H}$ and vectors of $D$ to be

$$
\left(C^{H}\right)_{i, *}=v^{H} Q_{\Gamma} J_{\Gamma}^{i-1} \widetilde{Q}_{\Gamma} .
$$

And $D_{i, *}=Q_{\Gamma} J_{\Gamma}^{i-1} \widetilde{Q}_{\Gamma} v$ for $i=1,2, \ldots, m$, respectively, we have $H=M_{0}$ and $H_{m}^{<}=M_{1}$. As for the rank of $\widetilde{Q}_{\Gamma} D$, we consider that column vectors of $\widetilde{Q}_{\Gamma} D$ from the Krylov series of $J_{\Gamma}$ starting from $\widetilde{Q}_{\Gamma} v$. Because $J_{\Gamma}$ has not degenerated, and elements of $\widetilde{Q}_{\Gamma} v$ are nonzero, these column vectors are linearly independent, and thus the rank of $\widetilde{Q}_{\Gamma} D$ is $f k_{\Gamma}$.

\section{Block Version of the SS Method with the Rayleigh-Ritz Procedure (BSSRR Method)}

We suggest a new algorithm for computing all poles of analytic function (2) with the use of the algorithm in [19]. As the eigenpairs $\left(\lambda_{i}, x_{i}\right)$ of equation (1) can be obtained from $H_{M}^{(1)} u_{i}=\theta_{i} H_{M}^{(2)} u_{i}$, where $H_{M}^{(1)}$ and $H_{M}^{(2)}$ are small $M \times M$ Hankel matrices. Let $V \in \mathbb{C}^{n \times l} \backslash\{0\}$, a random matrix, and $S=\left[S_{0}, S_{1}, \ldots, S_{M-1}\right] \in \mathbb{C}^{n \times L M}$, where 


$$
\begin{aligned}
S_{k} & =\left[s_{k}^{(1)}, \ldots, s_{k}^{(L)}\right] \\
& =\frac{1}{2 \pi i} \oint_{\Gamma}^{1}(z-\gamma)^{k}(z B-A)^{-1} \mathrm{BVd}, \quad k=0,1, \ldots, M-1 .
\end{aligned}
$$

Then, the block version of the SS method with Rayliegh-Ritz procedure [20] constructs the LM-dimensional subspace:

$$
\delta_{M}=\operatorname{span}\{S\}=\operatorname{span}\left\{s_{0}^{(1)}, \ldots, s_{0}^{(L)}, s_{1}^{(1)}, \ldots, s_{1}^{(L)}, \ldots, s_{M-1}^{(1)}, \ldots, s_{M-1}^{(L)}\right\} .
$$

For the Rayleigh-Ritz procedure, the subspace $\delta_{M}$ contains all eigenvectors of (1) $\delta_{M}=\operatorname{span}\left\{x_{1}, x_{2}, \ldots, x_{m}\right\}$ for $m \leq \mathrm{LM}$. With using $\mathrm{N}$-point trapezoidal rule for equation (25), we have

$$
S_{k} \approx \widetilde{S}_{k}=\sum_{j=1}^{N} \omega_{j}\left(z_{j}-\gamma\right)^{k}\left(z_{j} B-A\right)^{-1} \mathrm{BV} .
$$

where $z_{j}$ is the quadrature point and $\omega_{j}$ is the corresponding weight [21].

Based on Theorem 4, we analyse the relationship between the contour integral spectral projection and the Krylov subspace.
5.1. An Arnoldi-Based Interpretation of the Contour Integral Spectral Projection. Since the matrices $\widetilde{P}, Q$ are nonsingular, we define $P=\widetilde{P}^{-1}, \widetilde{Q}=Q^{-1}$. According to the Jordan block structure of (18), we partition row vectors in $\widetilde{P}, \widetilde{Q}$ into $\widetilde{P}_{i}, \widetilde{Q}_{i} \in \mathbb{C}^{n_{i} \times n}$ and column vectors in $P, Q$ into $P_{i}, Q_{i} \in \mathbb{C}^{n \times n_{i}}$, respectively, for $i=1,2, \ldots, r$. Then, we can derive the following lemma and theorem.

Lemma 1. Let $\psi_{k}(z)$ be a $k$-degree polynomial. Then, we have

$$
\frac{1}{2 \pi i} \oint_{\Gamma}^{1} \psi_{k}(z-\gamma)(z B-A)^{-1} B \mathrm{~d} z=\psi_{k}\left(S_{\Gamma}\right) P_{\Gamma},
$$

where

$$
S_{\Gamma}=\sum_{i: \lambda_{i} \in \Omega} Q_{i}\left(J_{i}-\gamma I\right) \widetilde{Q}_{i}, P_{\Gamma}=\sum_{i: \lambda_{i} \in \Omega} Q_{i} \widetilde{Q}_{i} .
$$

Proof. From Theorem 6 and the binomial theorem $(z-\gamma)^{\ell}=\sum_{j=0}^{\ell}\left(\begin{array}{c}\ell \\ j\end{array}\right) z^{\ell-j} \gamma^{j}$, we have the following relation: $\frac{1}{2 \pi i} \oint_{\Gamma}^{1}(z-\gamma)^{\ell}(z B-A)^{-1} B \mathrm{~d} z=\sum_{i: \lambda_{i} \in \Omega} Q_{i}\left(J_{i}-\gamma I\right)^{\ell} \widetilde{Q}_{i}, \quad \ell=0,1, \ldots$

Here, since $\widetilde{Q}_{i} Q_{i}=I$ and $\widetilde{Q}_{i} Q_{j}=0$ for $i \neq j$,

$$
\sum_{i: \lambda_{i} \in \Omega} Q_{i}\left(J_{i}-\gamma I\right)^{\ell} \widetilde{Q}_{i}=\left(\sum_{i: \lambda_{i} \in \Omega} Q_{i}\left(J_{i}-\gamma I\right) \widetilde{Q}_{i}\right)^{\ell}\left(\sum_{i: \lambda_{i} \in \Omega} Q_{i} \widetilde{Q}_{i}\right)=S_{\Gamma}^{\ell} P_{\Gamma}, \quad \ell=0,1, \ldots
$$

Therefore, Lemma 1 is proved.

Definition 2. Let $A \in \mathbb{C}^{n \times n}, V=v_{1}, v_{2}, \ldots, v_{L} \in \mathbb{C}^{n \times L}$, and let $\kappa_{m}\left(A, v_{1}\right)$ the Krylov subspace defined by

$$
\kappa_{m}\left(A, v_{1}\right)=\operatorname{span}\left\{v_{1}, A v_{1}, \ldots, A^{m-1} v_{1}\right\}
$$

Then, the subspace $\kappa_{m}^{*}(A, V)$ is defined by the sum of the Krylov subspaces, i.e.,

$$
\begin{aligned}
\kappa_{m}^{*}(A, V) & =\kappa_{m}\left(A, v_{1}\right)+\kappa_{m}\left(A, v_{2}\right)+\cdots+\kappa_{m}\left(A, v_{L}\right) \\
& =\operatorname{span}\left\{\left[V, A V, \ldots, A^{m-1} V\right]\right\} .
\end{aligned}
$$

Theorem 10. Let $\delta_{m}$ be the subspace of the block version of the SS method with the Rayleigh-Ritz procedure defined by (26). Then, we have

$$
\delta_{m}=\kappa_{m}^{*}\left(S_{\Gamma}, P_{\Gamma} V\right) .
$$

Proof. From the definition of $S_{k}(25)$ and Lemma 1, we have $S_{k}=S_{\Gamma}^{k} P_{\Gamma} V$. Therefore,

$$
\begin{aligned}
\delta_{M} & =\operatorname{span}\left\{\left[S_{0}, S_{1}, \ldots, S_{k}\right]\right\} \\
& =\operatorname{span}\left\{\left[P_{\Gamma} V, S_{\Gamma} P_{\Gamma} V, \ldots, S_{\Gamma}^{M-1} P_{\Gamma} V\right]\right\}=\kappa_{M}^{*}\left(S_{\Gamma}, P_{\Gamma} V\right) .
\end{aligned}
$$

Therefore, Theorem 10 is proved.

Remark 1. Theorem 10 shows that the block version of the SS method with the Rayleigh-Ritz procedure can be regarded as the Rayleigh-Ritz procedure based on the block Krylov subspace $\kappa_{M}^{*}\left(S_{\Gamma}, P_{\Gamma} V\right)$. Here, we note that, in the block version of the SS method with the Rayleigh-Ritz procedure, the basis vectors of $\kappa_{M}^{*}\left(S_{\Gamma}, P_{\Gamma} V\right)$ are explicitly computed by (25) and the $\mathrm{QR}$ decomposition of $\mathrm{S}$ (Algorithm 1).

And $\left(\lambda_{i}, x_{i}\right)=\left(\theta_{i}, W u_{i}\right)$ for $i=1,2, \ldots, \mathrm{LM}$.

\section{Numerical Experiments}

In this section, we have provided five numerical examples. In Examples 1-4, we have discussed stability Algorithm 2 (SS method), Algorithm 3 (CIRR method), Algorithm 4 (BSS method), and Algorithm 1 (BSSRR method), and in Example 5 , we have applied the BSSRR method with selected matrices in the fields of engineering sciences. Also, we have used 
Input: $L, M, N \in \mathbb{N}, V \in \mathbb{C}^{n \times L}, \gamma,\left(z_{j}, \omega_{j}\right)$ for $j=1,2, \ldots, N$

Output: eigenpairs $\left(\lambda_{i}, x_{i}\right)$ for $i=1,2, \ldots, \mathrm{LM}$

(1) Solve $\left(z_{j} B-A\right) Y_{j}=\mathrm{BV}$ for $j=1,2, \ldots, N,[14-16]$

(2) Compute $S_{k}=\sum_{j=1}^{N} \omega_{j}\left(z_{j}-\gamma\right)^{k} Y_{j}$ for $k=0,1, \ldots, M-1$

(3) Compute QR decomposition of $S=\left[S_{0}, S_{1}, \ldots, S_{M-1}\right]: S=W R$

(4) Compute eigenpairs $\left(\theta_{i}, u_{i}\right)$ of the matrix pencil $\left(W^{H} A W, W^{H} B W\right)$

Algorithm 1: BSSRR method.

Input: $u, v \in \mathbb{C}^{n}, N, m, \gamma, \rho$

Output: $\hat{\lambda}_{1}, \ldots, \hat{\lambda}_{m}, \hat{q}_{1}, \ldots, \hat{q}_{m}$

(1) Set $\omega_{j} \longleftarrow \gamma+\rho(2 \pi i j / N), j=0,1, \ldots, N-1$

(2) Solve $\left(\omega_{j} B-A\right) y_{j}=v, j=0,1, \ldots, N-1,[14-16]$

(3) Set $f_{j} \longleftarrow u^{H} y_{j}, j=0,1, \ldots, N-1$

(4) Compute $\widehat{\mu}_{k}, k=0, \ldots, 2 m-1$ by (5)

(5) Compute the eigenvalues $\xi_{1}, \ldots, \xi_{m}$ of the pencil $\widehat{H}_{m}^{<}-\lambda \widehat{H}_{m}$

(6) Compute $\hat{q}_{1}, \ldots, \hat{q}_{m}$ by (7)

(7) Set $\hat{\lambda}_{j} \longleftarrow \gamma+\xi_{j}, j=1, \ldots, m$

Algorithm 2: SS method.

Input: $v \in \mathbb{R}^{n}, N, M, \gamma, \rho$

Output: $\widehat{\lambda}_{1}, \ldots, \widehat{\lambda}_{m}, \widehat{x}_{1}, \ldots, \widehat{x}_{m}$

(1) Set $\omega_{j}=\gamma+\rho \exp (2 \pi i(j+1 / 2) / N), j=0,1, \ldots, N-1$

(2) Solve $\left(\omega_{j} B-A\right) y_{j}=B v$ for $y_{j}, j=0,1, \ldots, N-1,[14-16]$

(3) Compute $\widehat{s}_{k}, k=0,1, \ldots, M-1$ by (17)

(4) Construct an orthonormal basis $Q$ from $\left\{\widehat{s}_{0}, \ldots, \widehat{s}_{M-1}\right\}$

(5) Form $\widetilde{A}=Q^{T} A Q$ and $\widetilde{B}=Q^{T} B Q$

(6) Compute the eigenpairs $\left(\theta_{j}, \omega_{j}\right)(1 \leq j \leq M)$ of $(\widetilde{A}, t \widetilde{B})$

(7) Set $p_{j}=Q \omega_{j}, j=1, \ldots, M$

(8) Select the approximate eigenpairs $\left(\widehat{\lambda}_{1}, \widehat{x}_{1}\right), \ldots,\left(\widehat{\lambda}_{m}, \widehat{x}_{m}\right)$ from $\left(\theta_{j}, p_{j}\right)(1 \leq j \leq M)$

Algorithm 3: CIRR method.

Input: $V \in \mathbb{C}^{N \times l},\left\{z_{j}, \omega_{j}\right\}$ for $j=1,2, \ldots, M$

Output: $\alpha_{k}$ for $k=1,2, \ldots, K$

(1) Solve $\left(z_{j} B-A\right) \widetilde{V}_{j}=B V[14-16]$ and calculate $V_{j}=V^{H} \widetilde{V}_{j} \in \mathbb{C}^{l \times l}$

(2) Compute $\bar{\mu}_{n}=\sum_{j=1}^{M} \omega_{j} z_{j}^{n} V_{j}$

(3) Construct Hankel matrices $H_{m}$ and $H_{m}^{<} \in \mathbb{C}^{m l \times m l}$

(4) Perform singular value decomposition, $H_{m}=W s U$

(5) Construct $H=s^{-1 / 2} W^{H} H_{m}^{<} U^{H} s^{-1 / 2} \in \mathbb{C}^{K \times K}$

(6) Compute eigenvalues of $H$ to have $\alpha_{k}$

(7) Compute $\bar{S}_{n}=\sum_{j=1}^{M} \omega_{j} z_{j}^{n} \widetilde{V}_{j}$

(8) Compute $\left(Q_{1}, \ldots, Q_{K}\right)=\left(\bar{S}_{0}, \ldots, \bar{S}_{m-1}\right) U^{H} s^{-1 / 2}\left(q_{1}, \ldots, q_{K}\right)$

Algorithm 4: BSS method.

$A x_{i}+\lambda_{i} B x_{i 2} /\left(A x_{i 2}+\left|\lambda_{i}\right| B x_{i 2}\right)$ to compute relative residual for all of the methods. In computational results tables, the number of eigenpairs has been named NE.

Example 1. A real symmetric matrix $A \in \mathbb{R}^{n \times n}$ was prepared, which has five primary eigenvalues $-12.03,-12.02,-12.01$,
$-12.00,-11.99$. In the range of $[-12.5,-11.5]$, other eigenvalues were taken randomly in the range of $[-40,40]$, and a random unitary matrix was prepared to construct A. An identity matrix was used for B. After applying Algorithms 1-4, we obtained numerical results that have been shown in Table 1. 
TABle 1: The minimum relative residual of Algorithms 1-4 in Example 1.

\begin{tabular}{|c|c|c|c|c|c|c|c|c|}
\hline \multicolumn{9}{|c|}{$\gamma=-0.2+0.6 i, \rho=0.5$} \\
\hline \multirow[t]{2}{*}{ N.A } & \multicolumn{2}{|c|}{$m=16, N=32$} & \multicolumn{2}{|c|}{$M=18, N=32 m=16$} & \multicolumn{2}{|c|}{$\begin{array}{c}M=32, m=4, l=4 \\
\text { Threshold }=1.1 \times 10^{-18}\end{array}$} & \multicolumn{2}{|c|}{$M=4, L=4, N=32$} \\
\hline & SS & $\mathrm{NE}$ & CIRR & $\mathrm{NE}$ & BSS & $\mathrm{NE}$ & BSSRR & $\mathrm{NE}$ \\
\hline$n=200$ & 0.82114 & 16 & $8.8577 E-008$ & 16 & $1.9902 E-008$ & 16 & $7.9051 E-016$ & 16 \\
\hline$n=400$ & 0.7919 & 16 & $3.2359 E-06$ & 16 & $5.1353 E-09$ & 16 & $9.8608 E-16$ & 16 \\
\hline$n=600$ & 0.8393 & 16 & $3.2571 E-05$ & 16 & $1.1753 E-07$ & 16 & $1.3489 E-15$ & 16 \\
\hline$n=800$ & 0.8665 & 16 & $8.8640 E-05$ & 16 & $2.6448 E-05$ & 16 & $1.6268 E-15$ & 16 \\
\hline$n=1000$ & 0.8508 & 16 & $7.7542 \mathrm{E} E--05$ & 16 & $2.7825 E-05$ & 16 & $2.2567 E-15$ & 16 \\
\hline$n=1500$ & 0.8671 & 16 & $5.6523 E-05$ & 16 & $8.7373 E-005$ & 16 & $1.9151 E-15$ & 16 \\
\hline
\end{tabular}

TABLE 2: The minimum relative residual of Algorithms 1-4 in Example 2.

\begin{tabular}{|c|c|c|c|c|c|c|c|}
\hline \multicolumn{8}{|c|}{$\gamma=-0.1+0.5 i, \rho=0.4$} \\
\hline \multirow[t]{2}{*}{ N.A, N.B } & \multicolumn{2}{|c|}{$m=16, N=32$} & \multirow{2}{*}{$\begin{array}{c}M=18, N=32 m=16 \\
\text { CIRR }\end{array}$} & \multicolumn{2}{|c|}{$\begin{array}{c}M=32, m=4, l=4 \\
\text { Threshold }=1.1 \times 10^{-14}\end{array}$} & \multicolumn{2}{|c|}{$M=4, L=4, N=32$} \\
\hline & SS & $\mathrm{NE}$ & & BSS & $\mathrm{NE}$ & BSSRR & $\mathrm{NE}$ \\
\hline$n=200$ & 0.9307 & 16 & - & $9.919 E-05$ & 16 & $6.1079 E-16$ & 16 \\
\hline$n=400$ & 0.89555 & 16 & - & $1.3066 E-04$ & 16 & $8.3165 E-16$ & 16 \\
\hline$n=600$ & 0.8926 & 16 & - & $6.6731 E-004$ & 16 & $9.03555 E-16$ & 16 \\
\hline$n=800$ & 0.8802 & 16 & - & $1.600 E-004$ & 16 & $8.6891 E-16$ & 16 \\
\hline$n=1000$ & 0.8749 & 16 & - & $3.2000-004$ & 16 & $1.2295 E-15$ & 16 \\
\hline$n=1500$ & 0.8432 & 16 & - & $1.6400 E-004$ & 16 & $1.9151 E-15$ & 16 \\
\hline
\end{tabular}

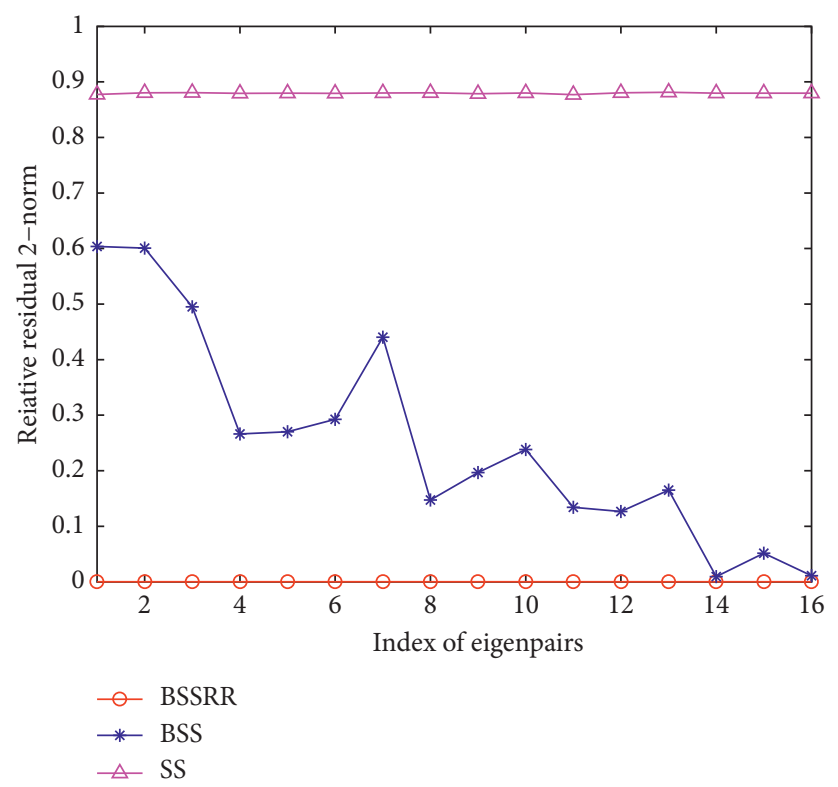

FIgURE 1: Results of comparing the relative residual of Algorithms 1-4 in Example 2 with $n=1000$.

TABLE 3: The minimum relative residual of Algorithm 1-4 and in Example 3.

\begin{tabular}{|c|c|c|c|c|c|c|c|c|}
\hline \multicolumn{9}{|c|}{$\gamma=0, \rho=0.14$} \\
\hline \multirow[t]{2}{*}{ N.A, N.B } & \multicolumn{2}{|c|}{$m=16, N=64$} & \multicolumn{2}{|c|}{$M=18, N=64 m=16 \backslash$} & \multicolumn{2}{|c|}{$\begin{array}{c}M=64, m=4, l=4 \\
\text { Threshold }=1.1 \times 10^{-18}\end{array}$} & \multicolumn{2}{|c|}{$M=4, L=4, N=64$} \\
\hline & SS & $\mathrm{NE}$ & CIRR & $\mathrm{NE}$ & BSS & $\mathrm{NE}$ & BSSRR & $\mathrm{NE}$ \\
\hline$n=200$ & 0.6600 & 16 & $5.7595 E-13$ & 16 & $3.4279 E-11$ & 8 & $6.3585 E-15$ & 16 \\
\hline$n=400$ & 0.6028 & 16 & $7.2639 E-11$ & 16 & $6.1907 E-11$ & 10 & $3.1440 E-15$ & 16 \\
\hline$n=600$ & 0.6509 & 16 & $3.4114 E-11$ & 16 & $3.0774 E-09$ & 11 & $3.3606 E-15$ & 16 \\
\hline$n=800$ & 0.5966 & 16 & $6.2826 E-11$ & 16 & $3.0774 E-08$ & 12 & $8.2243 E-15$ & 16 \\
\hline$n=1000$ & 0.6065 & 16 & $6.8571 E-10$ & 16 & $9.7887 E-08$ & 12 & $3.3916 E-15$ & 16 \\
\hline$n=1500$ & 0.6737 & 16 & $3.6078 E-12$ & 16 & $7.9782 E-08$ & 11 & $2.3400 E-15$ & 16 \\
\hline
\end{tabular}




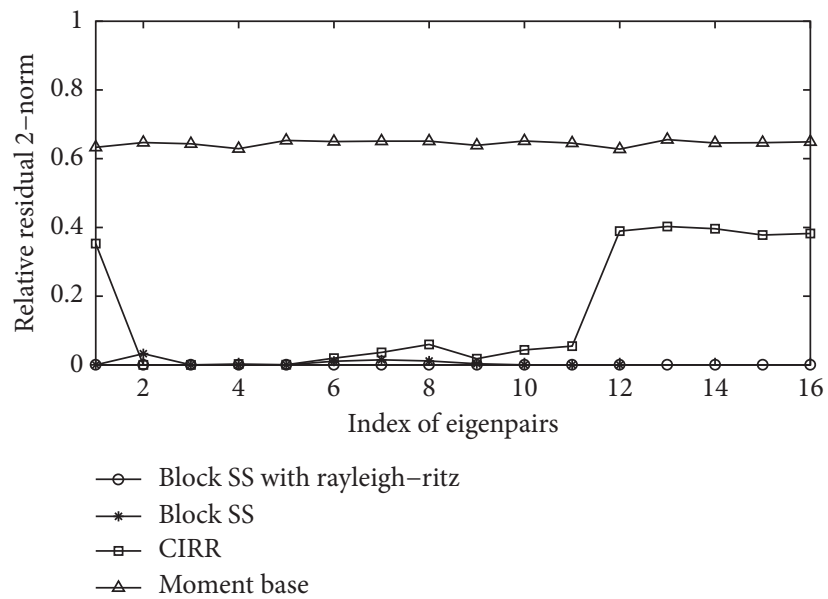

Figure 2: Results of comparing the relative residual of Algorithms 1-4 in Example 3 with $n=1000$.

TABLE 4: The minimum relative residual of Algorithms 1-4 in Example 4.

\begin{tabular}{|c|c|c|c|c|c|c|c|c|}
\hline \multicolumn{9}{|c|}{$\gamma=0, \rho=0.15$} \\
\hline \multirow[t]{2}{*}{ N.A, N.B } & \multicolumn{2}{|c|}{$m=16, N=32$} & \multicolumn{2}{|c|}{$\begin{array}{c}M=18, \\
N=32 m=16\end{array}$} & \multicolumn{2}{|c|}{$\begin{array}{c}M=32, m=4, l=4 \\
\text { Threshold }= \\
1.1 \times 10^{-14}\end{array}$} & \multicolumn{2}{|c|}{$M=4, L=4, N=32$} \\
\hline & SS & $\mathrm{NE}$ & CIRR & $\mathrm{NE}$ & BSS & $\mathrm{NE}$ & BSSRR & $\mathrm{NE}$ \\
\hline$n=200$ & 0.7389 & 16 & 0.0035 & 16 & 0.0315 & 16 & $2.4592 E-015$ & 16 \\
\hline$n=400$ & 0.7350 & 16 & 0.0057 & 16 & 0.0337 & 16 & $4.2091 E-15$ & 16 \\
\hline$n=600$ & 0.7370 & 16 & 0.0049 & 16 & 0.0349 & 16 & $5.2251 E-15$ & 16 \\
\hline$n=800$ & 0.7389 & 16 & 0.0068 & 16 & 0.0323 & 16 & $6.0494 E-15$ & 16 \\
\hline$n=1000$ & 0.7240 & 16 & 0.0069 & 16 & 0.0329 & 16 & $6.4196 E-15$ & 16 \\
\hline$n=1500$ & 0.7393 & 16 & 0.0065 & 16 & 0.0384 & 16 & $7.9371 E-15$ & 16 \\
\hline
\end{tabular}

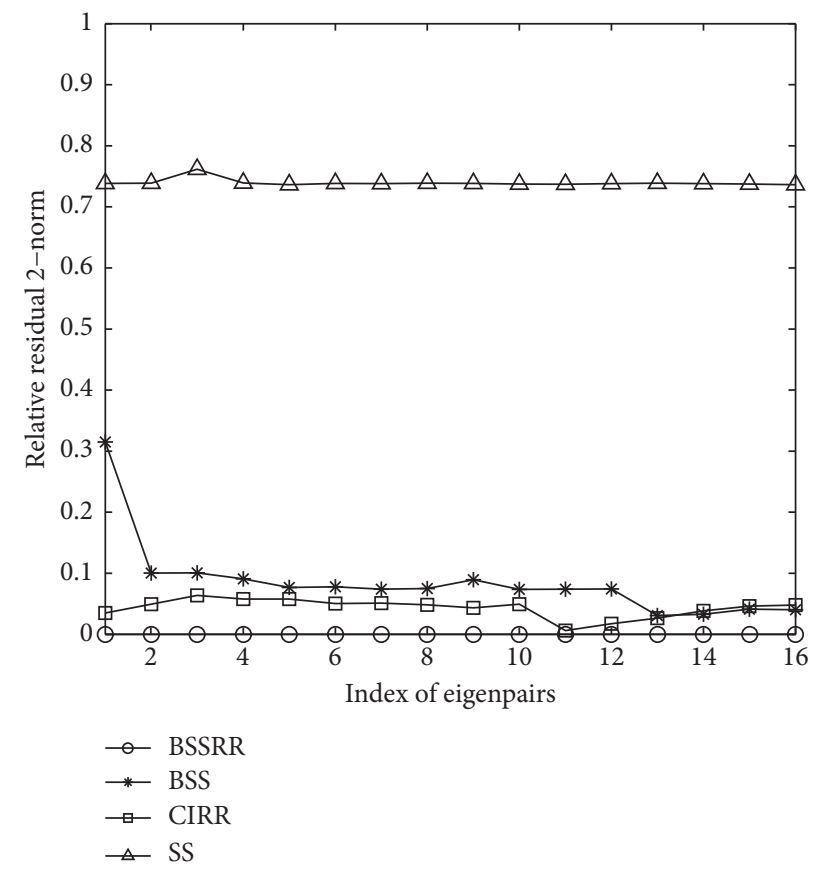

Figure 3: Results of comparing the relative residual of Algorithms 1-4 in Example 4 with $n=1000$. 
TABLE 5: Application areas, names, and properties 17 matrices selected.

\begin{tabular}{|c|c|c|c|c|}
\hline Application & Name & $\mathrm{N}$ & NZ & $\mathrm{CON}$ \\
\hline Generalized eigenvalue problem & lund_a & 147 & 2449 & $5.4430 E+006$ \\
\hline Generalized eigenvalue problem & lund_b & 147 & 2449 & $6.0317 E+004$ \\
\hline Chemical kinetics & fs_183_1 & 183 & 998 & $1.5122 E+013$ \\
\hline Square dielectric wave guide & dw256B & 512 & 2500 & 4.5047 \\
\hline Chemical kinetics & fs_760_1 & 760 & 5739 & $1.1234 E+016$ \\
\hline Large helicopter model & rotor 2 & 791 & 10685 & $1.2651 E+013$ \\
\hline Dynamic analysis in structural engineering & bcsstk19 & 817 & 6853 & $2.8130 E+011$ \\
\hline Unsymmetric basis from LP problem & bp_1600 & 822 & 4841 & $8.6511 E+006$ \\
\hline Power systems simulation & qh882 & 882 & 47906 & $9.9827 E+024$ \\
\hline Oil reservoir simulation & Sherman 1 & 1000 & 3750 & $2.2575 E+004$ \\
\hline Aeroelasticity & tols1090 & 1090 & 3546 & $2.1164 E+006$ \\
\hline Alfven spectra in magneto hydrodynamic & mdh1280 & 1280 & 47906 & $9.9827 E+024$ \\
\hline Nuclear reactor models & nnc261 & 1374 & 8588 & $4.1082 E+015$ \\
\hline Dynamic analysis in structural engineering & bcsstk12 & 1473 & 34241 & $5.2502 E+008$ \\
\hline Plasma physics & utm 1700 & 1700 & 21500 & $1.6380 E+007$ \\
\hline Splatzman symmetric finite difference three ocean model & Plat1919 & 1919 & 32399 & $5.1323 E+018$ \\
\hline Dynamic analysis in structural engineering & bcsstk13 & 2003 & 83883 & $4.5698 E+010$ \\
\hline
\end{tabular}

TABLE 6: Calculation relative residual 2-norm of the BSSRR method.

\begin{tabular}{|c|c|c|c|c|}
\hline \multicolumn{5}{|c|}{$\gamma=0, \rho=0.12, M=4, L=4, N=32$} \\
\hline \multicolumn{5}{|c|}{ BSSRR } \\
\hline Name matrix & $\mathrm{NE}$ & Minimum residual & Maximum residual & Time \\
\hline lund_a & 16 & $1.2115 E-015$ & $3.2651 E-015$ & 0.4351 \\
\hline lund_b & 16 & $7.7260 E-015$ & $4.9013 E-015$ & 0.4034 \\
\hline fs_183_1 & 16 & $9.4890 E-016$ & $7.6258 E-010$ & 0.6897 \\
\hline $\mathrm{dw} 256 \mathrm{~B}$ & 16 & $3.0480 E-015$ & $1.13825 E-013$ & 37.9230 \\
\hline fs_760_1 & 16 & $1.9950 E-015$ & $1.1748 E-014$ & 114.1690 \\
\hline rotor 2 & 16 & $2.9661 E-015$ & $7.6861 E-011$ & 73.6634 \\
\hline bcsstk19 & 16 & $1.7704 E-015$ & $4.3297 E-015$ & 80.9064 \\
\hline bp_1600 & 16 & $4.1415 E-015$ & $8.8643 E-015$ & 118.4747 \\
\hline qh882 & 16 & $8.3663 E-011$ & $8.1529 E-010$ & 91.1155 \\
\hline Sherman 1 & 16 & $2.0279 E-015$ & $6.7531 E-015$ & 145.8976 \\
\hline tols 1090 & 16 & $6.3521 E-15$ & $1.0472 E-12$ & 174.7583 \\
\hline mdh 1280 & 16 & $9.1528 E-015$ & $1.1804 E-012$ & 362.0046 \\
\hline nnc261 & 16 & $2.4955 E-015$ & $1.387 E-014$ & 362.3879 \\
\hline bcsstk12 & 16 & $1.7482 E-015$ & 4. $9099 E-015$ & 546.7578 \\
\hline utm 1700 & 16 & $5.0652 E-015$ & $2.6436 E-014$ & 960.8218 \\
\hline Plat1919 & 16 & $3.1237 E-015$ & $1.2013 E-013$ & 1191.3179 \\
\hline bcsstk13 & 16 & $2.8611 E-015$ & $5.3720 E-014$ & 1363.4479 \\
\hline
\end{tabular}

Example 2. We let that A, B were complex, random matrices, and B was positive definite. After applying Algorithms 1-4, we obtained numerical results that have been shown in Table 2. Also, the relative residual for described methods has been drawn in Figure 1 for $n=1000$.

Example 3. In this example, A, B were taken sparse, symmetric, and random, and A was positive definite. After applying Algorithms 1-4, we obtained numerical results that have been shown in Table 3 . Also, the relative residual for described methods has been drawn in Figure 2 for $n=1000$.

Example 4. We consider matrices:

$$
\begin{aligned}
A & =I_{n}, \\
B & =\left[\begin{array}{rrrrrrr}
-7 & -3 & -1 & -1 & -1 & -1 \\
-3 & -8 & -3 & -1 & -1 & -1 \\
-1 & -3 & -8 & -3 & -1 & -1 \\
-1 & \ddots & \ddots & \ddots & \ddots & \ddots \\
-1 & -1 & -3 & -8 & -3 & -1 \\
-1 & -1 & -1 & -3 & -8 & -3 \\
-1 & -1 & -1 & -1 & -3 & -7
\end{array}\right],
\end{aligned}
$$

After applying Algorithms 1-4, we obtained numerical results that have been shown in Table 4 . Also, the relative residual for described methods has been drawn in Figure 3 for $n=1000$. 
TABLE 7: Calculation minimum relative residual of the BSSRR method with other norms.

\begin{tabular}{|c|c|c|c|c|c|c|}
\hline \multicolumn{7}{|c|}{$\gamma=0, \rho=0.12, M=4, L=4, N=32$} \\
\hline \multicolumn{7}{|c|}{ BSSRR } \\
\hline Name matrix & $\|-\|_{1}$ & Time & $\|-\|_{\infty}$ & Time & $\|-\|_{\text {fro }}$ & Time \\
\hline lund_a & $1.6280 E-15$ & 0.4641 & $1.464 E-015$ & 0.4751 & $1.0893 E-015$ & 0.4429 \\
\hline lund_b & $1.2158 E-015$ & 0.4329 & $1.1523 E-015$ & 0.4289 & $9.5626 E-016$ & 0.4587 \\
\hline fs_183_1 & $1.3389 E-015$ & 0.9160 & $1.9050 E-015$ & 0.9926 & $5.7331 E-015$ & 0.7920 \\
\hline $\mathrm{dw} 256 \mathrm{~B}$ & $1.0643 E-014$ & 38.3247 & $4.8503 E-015$ & 38.5182 & $4.8285 E-015$ & 39.0655 \\
\hline fs_760_1 & $1.4451 E-014$ & 115.7634 & $5.8661 E-015$ & 116.8876 & $9.4203 E-015$ & 116.0084 \\
\hline rotor 2 & $8.5274 E-015$ & 74.3794 & $3.6814 E-015$ & 79.6705 & $1.5234 E-015$ & 75.7969 \\
\hline bcsstk19 & $4.6544 E-015$ & 83.6374 & $1.1299 E-015$ & 84.1706 & $1.6893 E-015$ & 83.6942 \\
\hline bp_1600 & $5.6919 E-15$ & 119.6776 & $2.1991 E-015$ & 119.9996 & $3.3914 E-015$ & 123.9625 \\
\hline qh882 & $2.7482 E-010$ & 98.5387 & $1.8575 E-010$ & 93.9466 & $1.0929 E-010$ & 94.3459 \\
\hline Sherman 1 & $2.2421 E-014$ & 148.6958 & $9.1555 E-015$ & 147.3571 & $2.1974 E-015$ & 160.7665 \\
\hline tols1090 & $6.4706 E-14$ & 203.0137 & $7.8958 E-14$ & 186.5922 & $8.0820 E--14$ & 175.2136 \\
\hline mdh 1280 & $1.1013 E-014$ & 357.0195 & $3.8742 E-015$ & 363.0094 & $6.2742 E-015$ & 357.0245 \\
\hline nnc261 & $2.8400 E-015$ & 391.5121 & $2.9017 E-015$ & 369.4831 & $3.2134 E-015$ & 363.7672 \\
\hline bcsstk12 & $1.0533 E-014$ & 615.0851 & $8.1737 E-015$ & 624.6021 & $1.8975 E-015$ & 582.1993 \\
\hline utm 1700 & $1.1256 E-14$ & 985.1695 & $6.1700 E-015$ & 1031.4640 & $5.5940 E-015$ & 968.1327 \\
\hline Plat1919 & $6.6578 E-015$ & 1173.1310 & $4.1980 E-015$ & 1294.0850 & $5.8991 E-015$ & 1296.4698 \\
\hline bcsstk13 & $7.4671 E-015$ & 1366.7534 & $3.5188 E-015$ & 1408.8137 & $3.2373 E--015$ & 1385.8719 \\
\hline
\end{tabular}

Example 5. In this example, we selected seventeen matrices from the UF sparse matrix collection. Two major requirements were used in the selection procedure: matrices with different parameters and matrices arising in different application areas were chosen. We consider the following symptoms parameters.

The order N, the number of nonzero elements NZ, and the condition number CON. The application areas of the selected matrices are listed in Table 5. Matrices from the different areas were selected and thus obtained results by running the matrices will be typical in several scientific fields. We applied the BSSRR method for the calculation of relative residual generalized eigenvalue problem (1) when $\mathrm{A}$ is one of the selected matrices in Table 5 and $\mathrm{B}$ is identity matrix. As the dimension A, B is equal. We computed relative residual respect to $-{ }_{2}$ In Table 6 and computed respect to other norms in Table 7 , too, the number of eigenpairs for each matrix was sixteen in Table 7.

\section{General Conclusions and Plans for Future Work}

Several specific conclusions were drawn in connection with the numerical results presented in the previous section. Some general conclusions are given as follows:

(1) All numerical experiments indicate that CIRR, BSS, and BSSRR methods have higher stability than respect to the SS method

(2) BSSRR method has less relative residual respect to SS, CIRR, and BSS methods

(3) If $-{ }_{2}$ is used for calculation of relative residual in the BSSRR method, then we have higher accuracy and less consuming time
Designing quadrature points with higher performance and a more precise error analysis of the BSSRR method is a part of our future work.

\section{Data Availability}

No data were used to support this study.

\section{Conflicts of Interest}

The authors declare that they have no conflicts of interest.

\section{Acknowledgments}

The support of Eng. Akbar Shahidzadeh Arabani is gratefully acknowledged.

\section{References}

[1] Z. Bai, "Krylov subspace techniques for reduced-order modeling of large-scale dynamical systems," Applied Numerical Mathematics, vol. 43, no. 1-2, pp. 9-44, 2002.

[2] Y. Inadomi, T. Nakano, K. Kitaura, and U. Nagashima, "Definition of molecular orbitals in fragment molecular orbital method," Chemical Physics Letters, vol. 364, no. 1-2, pp. 139-143, 2002.

[3] M. M. Magolu, "Incomplete factorization-based preconditions for solving the Helmholtz equation," International Journal for Numerical Methods in Engineering, vol. 50, pp. 1088-1101, 2001.

[4] K. Karthikeyan, "Small-signal stability enhancement using STATCOM based eigenvalue analysis," Indian Journal of Science Technology, vol. 21, 2015.

[5] J. Saira Banu, R. Babu, and R. Pandey, "Parallel implementation of singular value decomposition (SVD) in image compression using open $\mathrm{Mp}$ and sparse matrix representation," Indian Journal of Science Technology, vol. 35, 2015. 
[6] S. Gudarzi, H. H. Wan, M. H. Anisi, and A. Soleymani, "A comparative review of vertical handover decision-making mechanisms in heterogeneous wireless networks," Indian Journal of Science Technology, vol. 58, 2015.

[7] M. Kaviarasan, P. Geetha, and K. P. Soman, "Multivariative statical technique for the assessment of groundwater quality in Coonoor taluk, Nilgiri district, Tamilnadu, India," Indian Journal of Science Technology, vol. 112, 2015.

[8] B. N. Datta, Numerical Linear Algebra and Applications, Brooks, New York, NY, USA, 2010.

[9] H. S. Najafi, A. Refahi, and M. Akbari, "Weighted FOMinverse vector iteration method for computing a few smallest (largest) eigenvalues of pair (A,B)," Applied Mathematics and Computation, vol. 192, no. 1, pp. 239-246, 2007.

[10] H. Saberi Najafi and A. H. Refahi, "FOM-inverse vector iteration method for computing a few smallest (largest) eigenvalues of pair (A, B)," Applied mathematics and computation, vol. 17, pp. 614-647, 2007.

[11] H. Saberi Najafi and A. Refahi, "A new restarting method in the Lanczos algorithm for generalized eigenvalue problem," Applied Mathematics and Computation, vol. 184, no. 2, pp. 421-428, 2007.

[12] F. Gantmacher, The Theory of Matrices, Chelsea, New York, NY, USA, 1959.

[13] T. Sakurai and H. Sugiura, "A projection method for generalized eigenvalue problems using numerical integration," Journal of Computational and Applied Mathematics, vol. 159, no. 1, pp. 119-128, 2003.

[14] H. S. Najafi, S. A. Edalatpanah, and A. H. Refahi Sheikhani, "Convergence analysis of modified iterative methods to solve linear systems," Mediterranean Journal of Mathematics, vol. 11, no. 3, pp. 1019-1032, 2014.

[15] H. Saberi Najafi, S. A. Edalatpanah, and A. H. Refahisheikhani, "An analytical method as a preconditioning modeling for systems of linear equations," Computational and Applied Mathematics, vol. 37, no. 2, pp. 922-931, 2018.

[16] F. Shariffar, A. H. Refahi Sheikhani, and H. Saberi Najafi, "An efficient Chebyshev semi-iterative method for the solution of large systems," UPB Scientific Bulletin, Series A: Applied Mathematics and Physics, vol. 80, no. 4, pp. 239-252, 2018.

[17] T. Sakurai and H. Tadano, "CIRR: a Rayleigh-Ritz type method with contour integral for generalized eigenvalue problems," Hokkaido Mathematical Journal, vol. 36, no. 4, pp. 745-757, 2007.

[18] T. Ikegami, T. Sakurai, and U. Nagashima, "A filter diagonalization for generalized eigenvalue problems based on the sakurai - sugiura projection method," Journal of Computation and Applied Mathematics, vol. 41, pp. 1927-1936, 2010.

[19] P. Kravanja, T. Sakurai, and M. Van Barel, "On locating clusters of zeros of analytic functions," Bit Numerical Mathematics, vol. 39, no. 4, pp. 646-682, 1999.

[20] T. Ikegami and T. Sakurai, "Contour integral eigensolver for non-hermitian systems: a Rayleigh-ritz-type Approach," Taiwanese Journal of Mathematics, vol. 14, no. 3A, pp. 825$837,2010$.

[21] A. Imakura, L. Du, and T. Sakurai, "A block Arnoldi-type contour integral spectral projection method for solving generalized eigenvalue problems," Applied Mathematics Letters, vol. 32, pp. 22-27, 2014. 